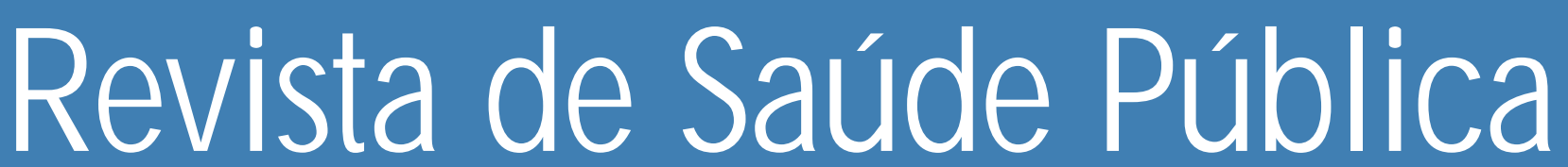

\begin{tabular}{lllllll}
\hline & 0 & $U$ & $R$ & $N$ & $A$ & $L$
\end{tabular}

$0 F$

P U B L I C

H E A L T H

\title{
Caracterização epidemiológica da doença meningocócica na área metropolitana do Rio de Janeiro, Brasil, 1976 a 1994
}

\section{Epidemiological characterization of meningococcal disease in a metropolitan area in Southeastern Brazil, 1976-1994}

Silvana Granado N. Gama, Keyla B. Feldman Marzochi e Getúlio Borges da Siveira Filho Escola Nacional de Saúde Pública da Fundação O swaldo Cruz. Rio de Janeiro, RJ - Brasil (S.G.N.G., K.B.F.M.), Faculdade de Economia e Administração da Universidade Federal do Rio de Janeiro. Rio de Janeiro, RJ - Brasil (G.B.S.F.) 


\title{
Caracterização epidemiológica da doença meningocócica na área metropolitana do Rio de Janeiro, Brasil, 1976 a 1994
}

\section{Epidemiological characterization of meningococcal disease in a metropolitan area in Southeastern Brazil, 1976-1994}

\author{
Silvana Granado N. Gama, Keyla B. Feldman Marzochi e Getúlio Borges da Siveira Filho
}

Escola Nacional de Saúde Pública da Fundação O swaldo Cruz. Rio de Janeiro, RJ - Brasil (S.G.N.G., K.B.F.M.), Faculdade de Economia e Administração da Universidade Federal do Rio de Janeiro. Rio de Janeiro, RJ - Brasil (G.B.S.F.)

\begin{abstract}
Resumo
Introdução

A doença meningocócica (DM) continua merecendo avaliações quanto a sua multicausalidade endêmica e epidêmica e seu comportamento evolutivo, nos diferentes locais.

Material e Métodos

Partindo da padronização da investigação epidemiológica da DM no Município do Rio de Janeiro a partir da epidemia da década de 70, foram analisados 4.155 casos notificados de 1976 a 1994, através de estudo retrospectivo, descritivo e analítico, com base nas fichas de investigação epidemiológica da Secretaria Municipal de Saúde. Os testes utilizados para análise estatística foram: o $\chi^{2}$, o de Wilcoxon-Mann-Whitney e de Kruskal-Wallis.

Resultados O estudo resultou na definição de três períodos, classificados como pós-epidêmico (1976/79), endêmico (1980/86) e epidêmico (1987/94), diferenciados pelas taxas de incidência e pelo sorogrupo do meningococo predominante. As taxas de incidência médias por período no município foram, respectivamente, de 3,51; 1,67 e 6,53 casos/100.000 habitantes. Os sorogrupos A e C predominaram no período pós-epidêmico, o $\mathrm{B}$ e o A no endêmico e o B no epidêmico. A letalidade média praticamente não se modificou no decorrer do tempo, mas variou segundo o hospital de internação, tendo sido sempre menor no hospital estadual de referência em relação aos demais públicos e privados.
\end{abstract}

Conclusão

\begin{abstract}
As maiores taxas de incidência e letalidade corresponderam aos menores de um ano e o risco de adoecer foi maior no sexo masculino. Os maiores coeficientes de incidência tenderam a ocorrer nas mesmas áreas do município, nos três períodos epidemiológicos, e a população que reside em favelas teve um risco de adoecimento duas vezes maior.
\end{abstract}

Infecções meningocócicas, epidemiologia.

Correspondência para/Correspondence to: Silvana Granado N. Gama - Rua Leopoldo Bulhões, 1480 - Sala 808 Manguinhos - $21041-210$ Rio de Janeiro, RJ - Brasil. E-mail: granado@ensp.fiocruz.br

Recebido em 1.8.1996. Reapresentado em 28.11.1996. Aprovado em 29.1.1997. 


\section{Introduction}

Material and Method

Results

Conclusion

\begin{abstract}
Meningococcal disease continues to warrant assessment as to its endemic and epidemic multicausality and temporal trends in various locations.
\end{abstract}

Based on a standardization of epidemiological investigation of meningococcal disease in the municipality of Rio de Janeiro county, Southeastern Brazil, as from epidemic of the 1970s a study to characterized the epidemiological characteristics of the disease, was realized. The total of 4,155 cases reported between 1976 and 1994 were analyzed in a retrospective, descriptive, and analytical study, using the epidemiological investigation forms issued by the Municipal Health Secretariat. Statistical analysis was performed using the $\chi^{2}$, Wilcoxon-Mann-Whitney, and Kruskal-Wallis tests.

The study resulted in the definition of three periods, classified as post-epidemic (1976-79), endemic (1980-86), and epidemic (1987-94), differentiated by the incidence rates and the predominant meningococcal serogroup. The mean incidence rates per period in the municipality were 3.51, 1.67, and 6.53 cases/ 100,000 inhabitants, respectively. Serogroups $A$ and $C$ predominated during the post-epidemic period, $B$ and $A$ in the endemic, and $B$ in the epidemic.

The mean case fatality rate remained virtually unchanged over time, but it varied by hospital, and during all three periods was lower in the State government reference hospital than in the other hospitals, whetter public or private. The highest incidence and case fatality rates were associated with patients under one year of age, and the risk of acquiring the disease was greater among males. The highest incidence coefficients tended to occur in the same areas of the conunty during the three epidemiological periods, and the shanty-town population was at twice the risk of acquiring the disease.

Meningococcal infections, epidemiology.

\section{INTRO DUÇÃO}

A doença meningocócica (DM) persiste como importante problema de Saúde Pública devido à sua magnitude, alta letalidade e, principalmente, por seu caráter endêmico-epidêmico. Além disto, trata-se de uma enfermidade cujo comportamento epidemiológico é ainda pouco conhecido, e contra a qual não se dispõe de medidas seguras de prevenção.

Na década de 80, a grande parte dos casos de DM registrados no mundo, com exceção a África, foram causados pelo sorogrupo B. Em países da Europa e EUA, esse sorogrupo vem se comportando quase sempre de forma endêmica. Porém, alguns países escandinavos, Holanda, Espanha e Irlanda, são exemplos de países desenvolvidos que vêm apresentando elevadas taxas de DM nos últimos anos (Peltola ${ }^{25}$, 1987; Schwartz e col. ${ }^{31}$, 1989; Fogarty e col. ${ }^{5}, 1994$; Scholten e col. $\left..^{30}, 1994\right)$. O meningococo do sorogrupo $\mathrm{C}$ vem sendo, mais recentemente, associado à ocorrência de surtos em diversos países, como EUA, Canadá, Israel e Brasil (Milagres e Melles ${ }^{19}$, 1993; Sacchi e col. ${ }^{28}$, 1994; Jackson e col. ${ }^{11}$, 1995; Grotto e col. ${ }^{9}, 1995$; Whalen e col. $\left.{ }^{35}, 1995\right)$.
Apesar da descoberta de novas vacinas, ainda se está longe de controlar a DM, principalmente os seus mecanismos de transmissão. Nem as vacinas polissacarídicas, nem a vacina protéica, ao que tudo indica, são eficazes seja, para impedir o estado de portador, seja para imunizar satisfatoriamente os pré-escolares, embora representando um indispensável avanço.

Assim sendo, a pretensão do presente estudo foi a de caracterizar o comportamento epidemiológico da doença meningocócica, nos anos de 1976 a 1994, no Município do Rio de Janeiro, definindo diferentes padrões epidemiológicos e determinando a incidência e letalidade por DM, conforme algumas características demográficas e sociais, e a qualidade de atenção médica prestada.

\section{MATERIAL E MÉTO DO}

O material básico de estudo procedeu das fichas de investigação epidemiológica domiciliar e hospitalar dos casos de DM notificados à Secretaria Municipal de Saúde (SMS) do Rio de Janeiro, no período de 1976 a 1994. O ponto de corte para início do estudo correspondeu ao ano 
em que a rotina de notificação e investigação epidemiológica dos casos se estabeleceu com um padrão de qualidade aceitável, visto que a vigilância epidemiológica da DM só teve início a partir de 1974, no decorrer da epidemia pelos sorogrupos $\mathrm{A}$ e $\mathrm{C}$.

As hipóteses que nortearam a busca das informações foram de que as taxas de incidência por DM vinham se comportando de forma desigual, no tempo e no espaço, e segundo o sorogrupo predominante.

Foi considerado como caso de DM aquele que estava de acordo com os critérios do Ministério da Saúde ${ }^{20}$ (1975), que é o adotado pelas Secretarias Municipal e Estadual de Saúde do Rio de Janeiro, ou seja, os que preencheram uma ou mais das seguintes condições: cultura do líquor positiva para meningococo; bacterioscopia positiva para diplococos Gram-negativos; contra-imunoeletroforese e/ou aglutinação pelo Látex positivos para meningococo A, B, C; citologia do líquor com celularidade aumentada e predomínio de neutrófilos associada a quadro clínico de exantema petequial; quadro clínico compatível com meningococcemia, confirmação por necrópsia; e referido como DM em atestado de óbito.

Com base na observação dos níveis de variação das taxas anuais de incidência, o período total de estudo, de dezenove anos, foi subdividido em três períodos. Para cada um foi calculada a taxa de incidência média — a soma de casos do período divididos pelo número de anos, sendo esta média dividida pela população mediana do período. As populações medianas corresponderam, respectivamente, aos anos de 1978, 1983 e 1991, para os períodos de 1976-1979, 1980-1986 e 1987-1994. O mesmo foi feito para os óbitos.

Os bairros foram agrupados segundo as Regiões Administrativas (RA) do Município do Rio de Janeiro. Segundo as taxas de incidência, as RAs foram classificadas em 4 faixas, distribuídas pelos períodos epidemiológicos. Para cada período, foram construídos mapas do município, distribuindo-se as RAs caracterizadas conforme a sua faixa, utilizando-se os pacotes SPANS map e SPANS GIS 5.2.

Pelo fato das RAs por vezes agruparem bairros socioeconomicamente heterogêneos, ou no mesmo bairro coexistirem favelas e residências de alto padrão, na tentativa de obter informação que melhor contribuísse para a caracterização socioeconômica, os casos foram também distribuídos em dois grupos: residentes em favelas ou áreas afins e em áreas urbanizadas.

Os hospitais foram agregados em 4 grupos: o primeiro correspondente exclusivamente ao Instituto Estadual de Infectologia São Sebastião (IEISS), como centro de referência para DM, no Estado do Rio de Janeiro; o segundo, incluindo todos os outros hospitais públicos, da rede municipal, estadual, federal, universitários ou militares; o terceiro, abrangendo toda a rede de hospitais privados ou conveniados, e o quarto grupo, cobrindo as situações excepcionais em que o paciente não chegou a ser internado.

Os indicadores utilizados na análise demográfica e social foram o sexo e a faixa etária, e o local de moradia (favela ou não), respectivamente. Para avaliar a qualidade da atenção médica prestada foi utilizada a letalidade média por grupos de hospitais.

Os dados populacionais foram obtidos a partir dos censos de 1970, 1980 e 1991. Para os anos intercensitários e para cada RA, as populações geral, por sexo e faixa etária foram estimadas com base na taxa anual média de crescimento do período entre os anos censitários de 1970/1980 e 1980/1991, via interpolação geométrica. Para estimativa dos anos pós censitários de 1992/94, admitiu-se válida a taxa de crescimento observada para o período 1980/1991.

Os testes utilizados para análises estatísticas (SPLUS $^{33}$, 1990), foram: a) Teste de quiquadrado, nas Tabelas 2 e 7, sendo que na Tabela 2, para a comparação duas a duas das distribuições dos números de casos por sorogrupo ao longo dos períodos, e na Tabela 7, para comparar as letalidades observadas nos três grupos de hospitais considerados. Foram desprezados eventuais problemas ligados à seletividade amostral. b) Teste de WilcoxonMenn-Whitney, na Tabela 6, a fim de se avaliar eventuais diferenças entre as letalidades por sexo; adicionalmente foi usado o teste de Kruskal-Wallis para comparação do perfil da letalidade por faixa etária ao longo dos 3 períodos considerados. c) Testes de Wilcoxon, para as Tabelas 3 e 4; na 3, para comparar as incidências segmentadas por faixa etária entre os períodos e, na 4, para comparar taxas de incidência segundo sexo e faixa etária.

\section{RESU LTAD O}

Foram levantados 4.155 casos de DM notificados entre 1976 e 1994. A observação dos níveis evolutivos de incidência anual permitiu caracterizar três períodos distintos: de 1976 a 1979 cuja variação da incidência média caiu de 5,50 para 2,95 por 100.000 habitantes - classificado como pós-epidêmico; de 1980 a 1986 quando essa taxa variou entre 1,08 e 2,63 casos/100.000 habitantes — endêmico; e de 1987 a 1994 cuja incidência foi de 4,23 a 9,24 casos/100.000 habitantes — epidêmico (Tabela 1).

A frequiência de realização de sorogrupagem dos casos notificados diferiu nos três períodos, sendo de $33 \%$ no primeiro, $22 \%$ no segundo e $49 \%$ no terceiro. No período pós epidêmico predominou o sorogrupo A $(63,4 \%)$ seguido pelo C $(31,7 \%)$; no período endêmico, enquanto se reduzia o A $(38,3 \%)$ crescia o B $(44,4 \%)$; e no período epidêmico o sorogrupo B $(80,1 \%)$ teve predomínio absoluto (Tabela 2$)$.

A idade dos pacientes variou entre um mês e 80 anos, porém cerca de $75 \%$ dos casos tinham menos de 15 anos, em todos os períodos. O grupo populacional mais atingido foi o das crianças menores de um ano, com acentuada diferença dos demais, revelando um risco quase 10 vezes maior de adquirir a doença do que a média geral (Tabela 3). 
Tabela 1 - Distribuição do número de casos e óbitos e taxas de incidência, de mortalidade e letalidade por doença meningocócica, segundo os períodos epidemiológicos. Município do Rio de Janeiro, 1976 - 1994.

Table 1 - Distribution of number of cases and deaths, incidence rates, mortality rates and case fatality rates for the meningococcal disease, for the epidemiological periods. Rio de Janeiro county, 1976-1994.

\begin{tabular}{lccccc}
\hline Período & Casos & $\begin{array}{c}\text { Tx incidência } \\
\text { média }\end{array}$ & Ó bitos & $\begin{array}{c}\text { Tx mortalidade } \\
\text { média }\end{array}$ & $\begin{array}{c}\text { Letalidade (\%) } \\
\text { média }\end{array}$ \\
\hline $1976-1979$ & 689 & 3,51 & 127 & 0,65 & 18,4 \\
$1980-1986$ & 604 & 1,67 & 98 & 0,27 & 16,2 \\
$1987-1994$ & 2.862 & 6,53 & 468 & 1,07 & 16,4 \\
\hline Total & 4.155 & $\ldots$ & 693 & $\ldots$ & 16,7 \\
\hline
\end{tabular}

Tabela 2 - Distribuição dos sorogrupos, segundo os períodos epidemiológicos. M unicípio do Rio de Janeiro, 1976 - 1994.

Table 2 - Distribution of serogroup, for the epidemiological periods. Rio de Janeiro county, 1976-1994.

\begin{tabular}{|c|c|c|c|c|c|c|c|c|}
\hline \multirow{3}{*}{ Período } & \multicolumn{6}{|c|}{ Sorogrupo } & \multirow{2}{*}{\multicolumn{2}{|c|}{ Total }} \\
\hline & \multicolumn{2}{|c|}{$---\underline{A}$} & \multicolumn{2}{|c|}{$B$} & \multicolumn{2}{|c|}{ C } & & \\
\hline & & $\%$ & $\overline{\mathrm{N}}$ & $\%$ & $\mathrm{~N}$ & $\overline{\%}$ & $\overline{\mathrm{N}}$ & $\%$ \\
\hline 1976-1979* & 142 & 63,4 & 11 & 4,9 & 71 & 31,7 & 224 & 100,0 \\
\hline $1980-1986$ & 51 & 38,3 & 59 & 44,4 & 23 & 17,3 & 133 & 100,0 \\
\hline $1987-1994 * *$ & 16 & 1,1 & 1.122 & 80,1 & 263 & 18,8 & 1.401 & 100,0 \\
\hline Total & 209 & 11,9 & 1.192 & 67,8 & 357 & 20,3 & 1.758 & 100,0 \\
\hline
\end{tabular}

$* p=0,001$ quando comparado ao período endêmico

$* * p=0,001$ quando comparado ao período endêmico

Tabela 3 - Distribuição das taxas de incidência por faixa etária, segundo os períodos epidemiológicos. Município do Rio de Janeiro, 1976 - 1994.

Table 3 - Distribution of incidence by age-group, for the epidemiological periods. Rio de Janeiro county, 1976-1994.

\begin{tabular}{lcccccc}
\hline \multirow{2}{*}{ Períodos } & \multicolumn{9}{c}{ Faixa Etária } & \multirow{2}{*}{ Total } \\
\cline { 2 - 6 } & $<1$ ano & $1-4$ & $5-9$ & $10-14$ & 15 e + & $3,51^{*}$ \\
$1976-1979$ & 28,73 & 10,89 & 7,40 & 3,57 & 1,57 & 1,67 \\
$1980-1986$ & 16,06 & 7,33 & 3,42 & 2,22 & 0,48 & $6,52^{* *}$ \\
$1987-1994$ & 60,07 & 32,26 & 16,40 & 7,94 & 1,94 & \\
\hline
\end{tabular}

$* p=0,03$ quando comparado ao período endêmico

** $p=0,03$ quando comparado ao período endêmico

Verificou-se um risco de adoecer maior no sexo masculino. Quando se considerou as respectivas populações por faixa etária, pôde-se verificar que, em todas as faixas e em cada período, a população masculina foi a mais acometida. Fez exceção apenas o grupo de 10 a 14 anos cujas taxas são semelhantes nos segundo e terceiro períodos entre os sexos (Tabela 4).

A distribuição das taxas médias de incidência por RA permitiu a classificação em quatro faixas de variação por período: RAs com taxas entre 0 e 2,99 casos/100.000 - primeira faixa; RAs com taxas entre 3,00 e 5,99 casos/100.000 - segunda faixa; RAs com taxas entre 6,00 e 8,99 casos/100.000 - terceira faixa e RA's com taxas iguais ou superiores a 9,00 casos/100.000 habitantes - quarta faixa.

Ao serem distribuídas as taxas nos mapas em cada período, apesar da mistura de altas e baixas incidências, verificou-se uma tendência à formação de "bolsões" da DM no município. No período pós-epidêmico (Figura 1), nenhuma RA alcançou valores da quarta faixa, isto é, taxa superior a 8,99 casos/100.000 e apenas duas RAs (I e XII) atingiram níveis correspondentes à terceira faixa $(6,00-8,99)$. No período endêmico $(\mathrm{Fi}-$ gura 2), não houve diferenças nos coeficientes médios entre as RAs, correspondentes em todas elas à primeira faixa, exceto a RA XXI que constitui a Ilha de Paquetá, a qual, com apenas 2 casos em 7 anos gerou uma taxa média de 10,5 casos/100.000 habitantes devido à pequena população; portanto, feita a exceção, nenhuma RA atingiu a 3 casos/ 100.000 no período endêmico. No período epidêmico (Figura 3) nenhuma RA apresentou coeficiente médio correspondente à primeira faixa $(<3$ casos/100.000) mas, ao contrário, 70,8\% das RAs apresentaram níveis correspondentes às faixas mais altas, terceira e quarta, portanto, com uma incidência predominante acima de 
Tabela 4 - Distribuição das taxas de incidência por faixa etária e sexo, segundo os períodos epidemiológicos. Município do Rio de Janeiro, 1976 - 1994.

Table 4 - Distribution of incidence rates by sex and age-groups, for the epidemiological periods. Rio de Janeiro county, 1976-1994.

\begin{tabular}{|c|c|c|c|c|c|c|}
\hline \multirow{3}{*}{ Idade / Sexo } & \multicolumn{6}{|c|}{ Período } \\
\hline & \multicolumn{2}{|c|}{1976 - 1979* } & \multicolumn{2}{|c|}{$1980-1986$} & \multicolumn{2}{|c|}{ 1987 - 1994* } \\
\hline & Masc & Fem. & Masctt. & Fem. & Masctt†. & Fem. \\
\hline$<1$ ano & 30,97 & 26,41 & 18,08 & 13,98 & 63,60 & 56,45 \\
\hline 1-4 anos & 11,81 & 9,94 & 7,92 & 6,73 & 37,55 & 26,85 \\
\hline 5-9 anos & 8,94 & 5,84 & 3,59 & 3,25 & 18,25 & 14,52 \\
\hline 10-14 anos & 4,98 & 2,18 & 2,31 & 2,13 & 7,90 & 8,00 \\
\hline 15 e + anos & 2,31 & 0,94 & 0,57 & 0,42 & 2,20 & 1,73 \\
\hline Total & 4,54 & 2,57 & 1,91 & 1,45 & 7,63 & 5,54 \\
\hline
\end{tabular}

$* p=0,003$ quando comparado com o período endêmico

** $p=0,003$ quando comparado com o período endêmico

$\dagger p=0,03$ quando comparado com o sexo feminino

t† $p=0,03$ quando comparado com o sexo feminino

t† $\mathrm{p}=0,052$ quando comparado com o sexo feminino

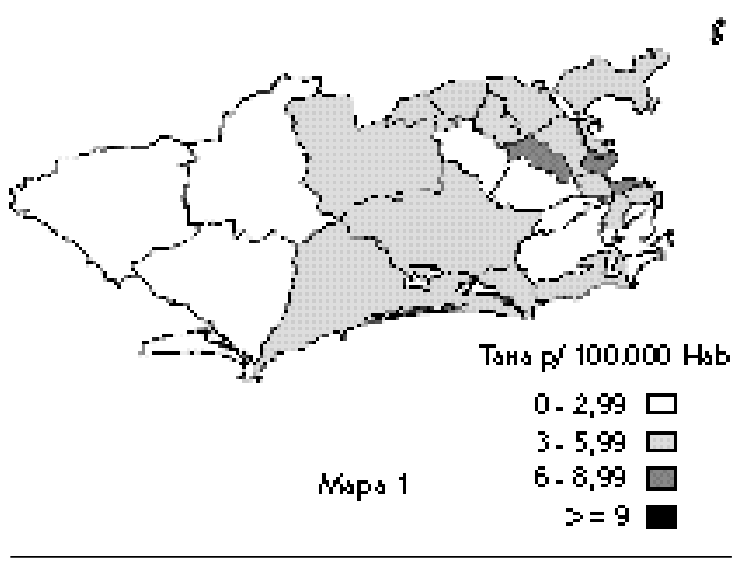

Figura 1 - Incidência da doença memingocócica por Região Administrativa no Município do Rio de Janeiro, 1976-1979.

Figure 1 - Indidence rates of meningocococcal disease in the Rio de Janeiro conty 1976-1979.

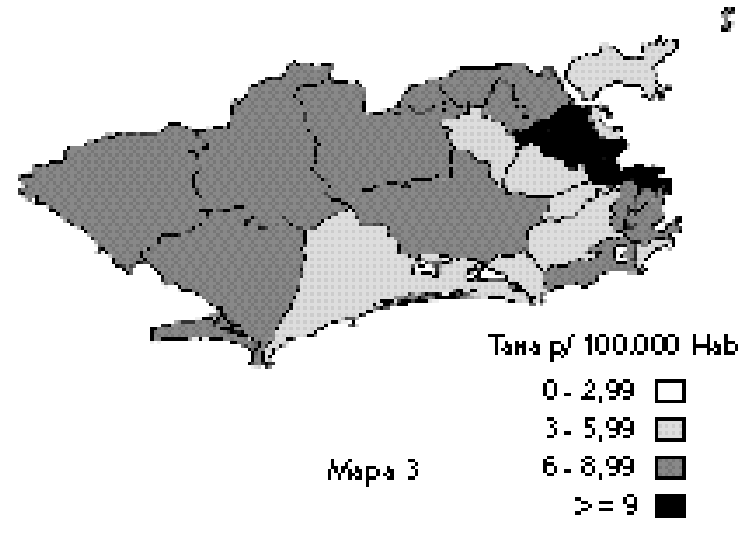

Figura 3 - Incidência da doença memingocócica por Região Administrativa no Município do Rio de Janeiro, 1987-1994.

Figure 3 - Indidence rates of meningocococcal disease in the Rio de Janeiro conty 1987-1994.

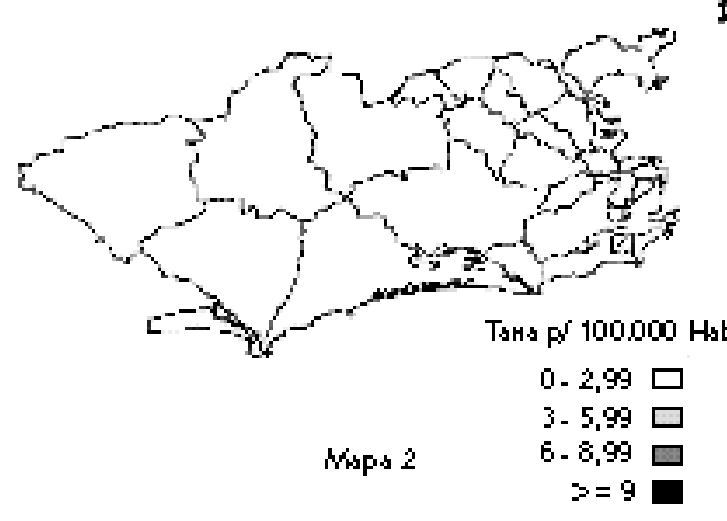

Figura 2 - Incidência da doença memingocócica por Região Administrativa no Município do Rio de Janeiro, 1980-1986.

Figure 2 - Indidence rates of meningocococcal disease in the Rio de Janeiro conty 1980-1986.

6 casos/100.000. As regiões de maior incidência $(\geq 9 /$ 100.000), que abrangeram as RAs I, II, VII, X e XII, são limítrofes geograficamente, além de concentrarem grande proporção de favelas ou áreas afins.

Quanto à variável tipo de local de moradia, obteve-se a informação em $70 \%$ dos casos notificados. Destes, $37,0 \%$ residiam em favelas. Entretanto, a incidência de casos de DM por população exposta é que definiu melhor essa relação. Por só dispor-se da informação da população favelada para o ano de 1991 (IBGE $\left.{ }^{6}, 1992 / 1993\right)$, calculou-se a taxa apenas para o terceiro período. Verificou-se assim que a taxa de incidência por DM nos favelados foi mais que o dobro da dos não favelados, 7,65 contra 3,71 por 100.000 hab. A discrepância entre as taxas acima pode ser devida a diferenças nas composições etárias das subpopulações envolvidas. Não foi possível ser 
mais conclusivo neste aspecto por não se dispor de estatísticas relativas à distribuição etária da população favelada ao longo do período considerado.

A grande maioria dos casos notificados de DM, $78,7 \%$, foram internados no IEISS, enquanto os restantes se dividiram de forma equilibrada entre os demais hospitais públicos e os privados.

As taxas de mortalidade seguiram o comportamento das de incidência, isto é, as maiores taxas no período epidêmico e as menores no período endêmico. Já as taxas de letalidade por DM, para o município como um todo, foram semelhantes duran- te as duas décadas estudadas. Nos menores de um ano ultrapassou $20 \%$ em todos os períodos, com diminuição progressiva até os 14 anos e discreto aumento a partir dos 15 anos. Apesar da incidência menor no sexo feminino, os óbitos tiveram peso ligeiramente maior neste grupo (Tabela 5). A letalidade, conforme hospital de atendimento, foi expressivamente menor no IEISS, em todos os períodos ( $\mathrm{Ta}$ bela 6), e dentre os que tiveram o sorogrupo identificado, a letalidade média foi mais elevada nos casos causados pelo sorogrupo A, $10,5 \%$; seguido pelo C, $8,9 \%$; e do $\mathrm{B}, \operatorname{com} 7,7 \%$.

Tabela 5 - Distribuição da letalidade média por faixa etária e por sexo, segundo os períodos epidemiológicos. Município do Rio de Janeiro, 1976 - 1994.

Table 5 - Distribution of average case fatality by age-groups and sex, for the epidemiological periods. Rio de Janeiro county, 1976-1994.

\begin{tabular}{|c|c|c|c|c|c|c|c|c|}
\hline \multirow{2}{*}{ Períodos* } & \multicolumn{5}{|c|}{ Faixa Etária** } & \multicolumn{2}{|c|}{ Sexo*** } & \multirow{2}{*}{ Total } \\
\hline & $<1$ ano & $1-4$ & $5-9$ & $10-14$ & $15 \mathrm{e}+$ & $\mathrm{M}$ asc. & Fem. & \\
\hline 1976-1979 & 31,9 & 19,0 & 12,2 & 11,3 & 17,0 & 17,9 & 19,4 & 18,4 \\
\hline $1980-1986$ & 24,5 & 16,5 & 9,3 & 10,1 & 17,8 & 14,6 & 18,2 & 16,2 \\
\hline 1987-1994 & 23,6 & 18,8 & 13,9 & 11,9 & 19,2 & 17,1 & 18,6 & 16,4 \\
\hline
\end{tabular}

* $p=0,67$ comparação entre os períodos

** $p=0,016$ comparação entre as faixas etárias

*** $p=0,0495$ comparação entre os sexos

Tabela 6 - Distribuição do número de casos, óbitos e das taxas de letalidade por doença meningocócica, segundo hospital de internação. Município do Rio de Janeiro, 1976-1994.

Table 6 - Distribution of number of cases, deaths and case fatality rate for the meningococcal disease, for the hospital of Rio de Janeiro county, 1976-1994.

\begin{tabular}{lccc}
\hline Hospitais & Número de casos & Número de óbitos & Letalidade (\%) \\
\hline IEISS & 2.509 & 275 & 11,0 \\
O utros públicos & 366 & 157 & 44,6 \\
Privados & 315 & 95 & 31,8 \\
\hline
\end{tabular}

$p=0,001$

$p=0,001$
IEISS - Instituto Estadual de Infectologia São Sebastião

\section{DISCU SSÃO}

O ponto de corte para caracterização de endemia foi selecionado com base em índices endêmicos da DM no Brasil e no exterior que, de regra, não atingem a 3 casos/100.000 habitantes, excluídos países africanos do chamado cinturão da meningite (Marzochi e col. ${ }^{17}, 1979$; Peltola ${ }^{25}$, 1987; Block e col. ${ }^{3}$, 1993). No Município do Rio de Janeiro ocorreu uma campanha de vacinação antimeningocócica BC no ano de 1990, cuja população alvo foram crianças entre 6 meses e 9 anos de idade, cujo efeito foi o de desacelerar, temporariamente, o crescimento da DM, inclusive em faixas etárias não contempladas com a vacina $\left(\mathrm{Gama}^{7}\right.$, 1995). Este estudo será detalhadamente discutido em próximo artigo através de técnicas de séries temporais.
Após a II Guerra Mundial, as epidemias tenderam a desaparecer dos EUA e da maioria dos países europeus, embora ainda ocorram ondas hiperendêmicas e surtos localizados. Na maioria desses países as taxas de incidência têm se mantido entre 1 e 2 casos por 100.000 habitantes, causados quase exclusivamente pelos sorogrupos B e C (Olyhoek e col. ${ }^{23}$, 1987; Pinner e col. ${ }^{26}$, 1991; Block e col ${ }^{3}, 1993$ ).

Maior concentração de casos em menores de 15 anos é uma característica da DM em quase todo o mundo, com exceção do cinturão africano da meningite onde a faixa predominante se desloca um pouco para cima, atingindo em maior proporção os escolares (Peltola $\left.{ }^{24}, 1983\right)$. O predomínio nas crianças pequenas tem sido reportado por vários autores (Tikhomirov $^{34}$, 1987; Fogarty e col.5. 1994). Majo ${ }^{14}$, 
1981, em Andaluzia na Espanha, entre os anos de 1956 e 1979, e Jones e Abbott ${ }^{12}$, 1987, na Inglaterra, nos anos de 1974 a 1983, verificaram que os menores de um ano correriam o maior risco de contrair esta enfermidade. Também Kemp ${ }^{13}$, 1994, encontrou o maior coeficiente em menores de um ano no $\mathrm{Mu}-$ nicípio de Campinas-SP. Por outro lado, Scholten e col. $.^{30}, 1994$, encontraram nos maiores de 50 anos a maior proporção de casos de DM, entre 1989 e 1990, na Holanda.

O predomínio da DM no sexo masculino já foi constatado por diversos autores (Bryan e col. ${ }^{4}, 1990$; Salih e col..$^{29}$, 1990; Fogarty e col. $\left.{ }^{5}, 1994\right)$, porém essa comparação tem sido feita, em geral, apenas pela proporção de casos e não por coeficientes, conforme se referiu. Pelos presentes dados, através dos coeficientes, verificou-se um risco maior para o sexo masculino em todos os períodos.

Quanto à distribuição espacial, também no Município do Rio de Janeiro, Nery-Guimarães e col. ${ }^{22}$, 1981, estudaram casos de meningite purulenta entre julho e dezembro de 1978, verificando maior incidência entre habitantes de favelas e conjuntos habitacionais populares. Marzochi ${ }^{18}$, 1985, em Londrina, PR, relacionou a maior ocorrência de DM a fatores socioeconômicos, durante epidemia, tais como baixa renda, tempo curto de moradia no mesmo bairro, analfabetismo e pirâmide etária com maior predomínio de crianças.

Moore e col. ${ }^{21}, 1990$, relacionaram a ocorrência de DM em refugiados no Sudão nos 6 primeiros meses de 1989 com maior risco de adoecer nesta população, associado às más condições de vida. $\mathrm{Na}$ África, Pinner e col. ${ }^{27}$, 1992, investigaram uma grande epidemia em Nairobi, Quênia, ocorrida entre 1989 e 1990, e distribuindo os casos por regiões da cidade, verificaram que as maiores concentrações corresponderam a áreas de grandes favelas. Por outro lado, Greenwood e col. ${ }^{8}, 1987$, estudaram fatores que influenciariam a suscetibilidade à DM durante epidemia pelo sorogrupo A, entre 1982/83, em Gambia, Oeste da África, onde fatores socioeconômicos, como aglomeração e estado nutricional, não foram considerados importantes.

Individualmente, a ausência de condições precárias de vida não garante a prevenção de casos, coletivamente, porém, a presença dessas condições favorece a instalação e difusão da doença; ou seja, pobreza e promiscuidade, embora sejam fatores importantes, não são suficientes para responderem isoladamente pela ocorrência da infecção clínica.

As taxas de mortalidade relativamente baixas no Rio de Janeiro respondem pela inserção pequena da
DM no conjunto dos óbitos, ao contrário da letalidade que se constitui um indicador epidemiológico importante no planejamento assistencial e preventivo. Esta última vem se apresentando sempre com valores médios elevados em comparação com a literatura. A alta letalidade em crianças pequenas é esperada, não só nas formas de meningococcemia, cuja gravidade independe da idade, mas também nos quadros de meningite ou mistos cujos prognósticos estariam associados à faixa etária (Martinez Torres ${ }^{15}$, 1994).

Em estudo de meta-análise, Baraff e col. ${ }^{1}$ (1993) reviram publicações após 1955 , onde são referidas taxas de letalidade por DM de diversos países, como por exemplo: México, 1983, 2,9\%; Canadá, 1986, 6,4\%; Nova Zelândia, 1988, 1,4\%; EUA, 1990, 1,8\% e 2,6\% (estudos distintos); Suíça, 1990, 7,0\%; Costa Rica, 1991, 2,0\%; Sudão, 1991, 25,0\%.

Quanto à diferença das taxas de letalidade por sorogrupo, obsevaram-se valores diferenciados na literatura. Spanjaard e col..$^{32}$, 1987, na Holanda, em estudo de associação do sorogrupo com o curso da DM encontraram valores bem inferiores aos verificados no Município do Rio de Janeiro para a letalidade por todos os sorogrupos: A - 2,3\%, B - 5,1\% e C - 4,8\%. Já Scholten e col. ${ }^{30}, 1994$, poucos anos depois (90/91) naquele mesmo país, identificaram letalidades mais altas, sendo $15,4 \%$ para o sorogrupo A, 5,6\% para o B e 12,4 para o C, valores bem mais próximos da nossa realidade, e que reforçam o achado de menores taxas no sorogrupo B. No Canadá, as taxas de letalidade por sorogrupo também se encontravam acima da média dos países desenvolvidos, com $6,0 \%$ para o grupo B e 14,5 para o grupo C (Whalen e col. ${ }^{35}$, 1995).

O IEISS apresentou uma qualidade de atendimento, dada pelas taxas de letalidade, visivelmente superior aos demais, fossem eles públicos ou privados. Não se sabe, porém, as reais condições de todos os outros serviços de saúde, a não ser dos hospitais universitários, que, através de seus respectivos setores de doenças infecciosas, devem garantir a qualidade do atendimento. Entretanto, o número de casos atendidos nos hospitais universitários foi relativamente pequeno, insuficiente para uma avaliação comparativa. No Rio de Janeiro, apenas o IEISS apresentou taxas de letalidade compatíveis com o registrado, em média, na literatura internacional, diferente da realidade da maioria dos demais. Embora a letalidade não dependa apenas da qualidade e rapidez no tratamento, mas também da própria imunidade do indivíduo e da virulência das cepas, certamente com a melhoria da qualidade do atendimento haveria menor número 
de óbitos. Com os avanços na antibioticoterapia e sofisticação de CTI's no tratamento da DM, as perdas se mantêm entre 5 e $10 \%$ dos casos nos países mais desenvolvidos, nas últimas décadas (Halstensen e col. ${ }^{10}, 1987$; Jones e Abbott ${ }^{12}$, 1987; Spanjaard e col. $^{32}$, 1987; Tikhomirov $^{34}$, 1987; Bjune e col. ${ }^{2}$, 1991; Scholten e col..$^{30}$, 1994; Whalen e col. $\left.{ }^{35}, 1995\right)$, enquanto no município estudado esses valores estão em torno de $17 \%$.

Concluindo, o estudo da DM no Município do Rio de Janeiro, ao longo de 19 anos permitiu a caracteri-

\section{REFERÊN CIAS BIBLIO G RÁFICAS}

1. BARAFF, L.J.; LEE, S.I.; SCHRIGER, D.L. Outcomes of bacterial meningitis in children: a meta-analysis. Pediatr. Infect. Dis. J., 12: 389-94, 1993.

2. BJUNE, G.; HOIBY, E.A.; GRONNESBY, J.K.; ARNESEN, O.; FREDRIKSEN, J.H.; HALSTENSEN, A.; HOLTEN, E.; LINDBAK, A.K.; NOKLEBY, H.; ROSENQVIST, E.; SOLBERG, L.K.; CLOSS, O.; ENG, J.; FROHOLM, L.O.; LYSTAD, A.; BAKKETEIG, L.S.; HAREIDE, B. Effect of outer membrane vesicle vaccine against group B meningococcal disease in Norway. Lancet, 338: 1093-6, 1991.

3. BLOCK, C.; ROITMAN, M.; BOGOCOWSKY, B.; MEIZLIN, S.; SLATER, P.E. Forty years of meningococcal disease in Israel: 1951-1990. Clin. Infect. Dis., 17: 126-32, 1993.

4. BRYAN, J.P.; SILVA, H.R.; TAVARES, A.; ROCHA, H.; SCHELD, W.M. Etiology and mortality of bacterial meningitis in Northeastern Brazil. Rev. Infect. Dis., 12: $128-35,1990$.

5. FOGARTY, J.; KEANE, C.T.; CARROLL, R.; BYRNE, M.; MOLONEY, A.C. Meningococcal disease in childhood - a regional study in Ireland. J. Infect., 28: 199-207, 1994.

6. FUNDAÇÃO IBGE. Anuário estatístico, 1991. Rio de Janeiro, 1993

7. GAMA, S.G.N. A doença meningocócica e sua evolução no Município do Rio de Janeiro - 1976 a 1994. Rio de Janeiro, 1995. [Dissertação de Mestrado - Escola Nacional de Saúde Pública/FIOCRUZ].

8. GREENWOOD, B.M.; GREENWOOD, A.M.; BRADLEY, K.; WILLIAMS, K.; HASSAN-KING, M.; SHENTON, F.C.; WALL, R.A.; HAYES, R.J. Factors influencing susceptibility to meningococcal disease during an epidemic in The Gambia, West Africa. J. Infect., 14: 167-84, 1987.

9. GROTTO, I.; BLOCK, C.; LERMAN, Y.; WIENER, M.; ASHKENAZI, S. Menigococcal disease in the Israel Defense Force: epidemiologic trends and new challenges. Isr. J. Med. Sci., 31: 54-8, 1995.

10. HALSTENSEN, A.; PEDERSEN, S.H.J.; HANERBERG, B.; BJORVATN, B.; SOLBERG, C.O. Case fatality of meningococcal disease in Western Norway. Scand. J. Infect. Dis., 19: 35-42, 1987. zação de três períodos epidemiológicos, sendo que, do período pós-epidêmico para o endêmico, houve um decréscimo na taxa de incidência média de $52,4 \%$, enquanto do período endêmico para o epidêmico a elevação foi de $291,0 \%$. A distribuição da DM pelas RAs parecem exibir regiões formadas por bairros vizinhos de taxas de incidência elevadas, favorecendo a hipótese de dependência espacial, apontando para a necessidade de maiores investigações com o auxílio de técnicas de detecção de aglomerados espaço -temporais, que será a próxima etapa de estudo.

11. JACKSON, L.A.; SCHUCHAT, A.; REEVES, M.W.; WENGER, J.D. Serogroup C meningococcal outbreaks in the United States. JAMA, 273: 383-9, 1995.

12. JONES, D.M. \& ABBOTT, J.D. Meningococcal disease in England and Wales. In: Vedros, N.A. Evolution of meningococcal disease. Boca Raton, CRC Press, Inc., 1987, v 1 , p.65-89.

13. KEMP, B. Aspectos epidemiológicos e diagnóstico laboratorial da doença meningocócica no Município de Campinas-SP no período de 1988 a 1993. São Paulo, 1994. [Dissertação de Mestrado - Faculdade de Saúde Pública da USP].

14. MAJO, M.C. Epidemiologia descriptiva de las meningitis en Andalucia. 1956-1979. Rev. San. Hig. Pública, 55: 665-8, 1981.

15. MARTINEZ-TORRES, E. Enfermidad menigococica: fisiopatologia, cuadro clinico y prognostico. Rev. Hosp. Niños. B. Aires, 36: 204-14, 1994.

16. MARZOCHI, K.B.F. Aspectos epidemiológicos da doença meningocócica no Município de Londrina, Paraná, no período de 1965-1975. Rio de Janeiro, 1977. [Dissertação de Mestrado - Faculdade de Medicina da Universidade Federal do Rio de Janeiro].

17. MARZOCHI, K.B.F.; CAMILLO-COURA, L.; MARZOCHI, M.C.A. Evolução histórica das epidemias da doença meningocócica. Ciênc. Cult., 31: 723-31, 1979.

18. MARZOCHI, K.B.F. Doença meningocócica epidêmica: avaliação de aspectos climáticos e socioeconômicos, de portadores e da ação vacinal, em município de médio porte do Sul do Brasil. Rio de Janeiro, 1985. [Tese de Doutorado Faculdade de Medicina da Universidade Federal do Rio de Janeiro]

19. MILAGRES, L.G. \& MELLES, C.E.A. Imunidade conferida por vacinas antimeningocócicas. Rev. Saúde Pública, 27: 221-6, 1993.

20. MINISTÉRIO DA SAÚDE. Secretaria Nacional de Saúde. Comissão Nacional de Controle de Meningite. Doença meningocócica. Brasília, 1975. 
21. MOORE, P.S.; TOOLE, M.J.; NIEBURG, P.; WALDMAN, R.J.; BROOME, C.V. Surveillance and control of meningococcal meningitis epidemics in refugee populations. Bull. WHO, 68: 587-96, 1990.

22. NERY-GUIMARÃES, R.; BITTENCOURT, L.C.M.; PASTOR, N.V.A. Meningites virais e bacterianas no Município do Rio de Janeiro (Brasil): algumas considerações sobre o sistema de informações em saúde e sobre a distribuição da doença no espaço urbano. Rev. Saúde Pública, 15: 379-94, 1981.

23. OLYHOEK, T.; CROWE, B.A.; ACHTMAN, M. Clonal population structure of Neisseria meningitidis serogroup A isolated from epidemics and pandemics between 1915 and 1983. Rev. Infect. Dis., 9: 665-92, 1987.

24. PELTOLA, H. Meningococcal disease: still with us. Rev. Infect. Dis., 5: 71-91, 1983.

25. PELTOLA, H. Meningococcal disease: an old enemy in Scandinavia. In: Vedros, N. A. Evolution of meningococcal disease. Boca Raton, CRC Press, Inc., 1987. v.1, p. 91-102.

26. PINNER, R.W.; GELLIN, B.G.; BIBB, W.F.; BAKER, C.N.; WEAVER, R.; HUNTER, S.B.; WATERMAN, S.H.; MOCCA, L.F.; FRASCH, C.E.; BROOME, C.V. Meningococcal disease in the United States-1986: Meningococcal Disease Study Group. J. Infect. Dis., 164: 368-74, 1991.

27. PINNER, R.W.; ONYANGO, F.; PERKINS, B.A.; MIRZA, N.B.; NGACHA, D.M.; REEVES, M.; DEWITT, W.; NJERU, E.; AGATA, N.N.; BROOME, C.V. Epidemic Meningococcal disease in Nairobi, Kenya, 1989: The Kenya Centers for Disease Control (CDC) Meningitis Study Group. J. Infect. Dis., 166: 359-64, 1992.
28. SACCHI, C.T.; TONDELLA, M.L.C.; LEMOS, A.P.S.; GORLA, M.C.O.; BERTO, D.B.; KUMIOCHI, N.H.; MELLES, C.E.A. Characterization of epidemic Neisseria meningitidis serogroup C strain in several Brazilian States. $J$. Clin. Microbiol., 32: 1783-7, 1994.

29. SALIH, M.A.M.; AHMED, H.S.; KARRAR, Z.A.; KAMIL, I.; OSMAN, K.A.; PALMGREN, H.; HOFVANDER, Y.; OLCÉN, P. Features of a large epidemic of group A meningococcal meningitis in Khartoum, Sudan in 1988. Scand. J. Infect. Dis., 22: 161-70, 1990.

30. SCHOLTEN, R.J.P.M.; BIJLMER, H.A.; VALKENBURG, H.A.; DANKERT, J. Patient and strain characteristics in relation to the outcome of meningococcal disease: a multivariate analysis. Epidemiol. Infect., 112: 115-24, 1994.

31. SCHWARTZ, B.; MOORE, P.S.; BROOME, C.V. Global epidemiology of meningococcal disease. Clin. Microbiol. Rev., 2: 118-24, 1989.

32. SPANJAARD, L.; BOL, P.; MARIE, S.; ZANEN, H.C. Association of meningococcal serogroups with the course of disease in the Netherlands, 1959-83. Bull WHO, 65: 861-8, 1987.

33. S-PLUS for DOS. Statistical Sciences, Inc., 1990.

34-TIKHOMIROV, E. Meningococcal meningitis: global situation and control measures. World Health Stat. Q., 40: 98-108, 1987.

35. WHALEN, C.M.; HOCKIN, J.C.; RYAN, A.; ASHTON, F. The changing epidemiology of invasive meningococcal disease in Canada, 1985 through 1992: emergence of a virulent clone of Neisseria meningitidis. JAMA, 273: 390-4, 1995. 